\title{
Creating Backup Management Resources for Spine Care during the Coronavirus Disease 2019 Pandemic
}

\author{
Sandeep Kumar Yadav ${ }^{1,2}$, Bikram Keshri Kar ${ }^{1}$, Aditya Banta ${ }^{3}$, Awkash Kumar ${ }^{4}$ \\ ${ }^{1}$ Department of Orthopedics, All India Institute of Medical Sciences, Raipur, India \\ ${ }^{2}$ Department of Trauma and Emergency, All India Institute of Medical Sciences, Raipur, India \\ ${ }^{3}$ Department of Spine Surgery, Shalby Hospital, Sahibzada Ajit Singh Nagar, India \\ ${ }^{4}$ Department of Orthopedics, Shalby Hospital, Ahmedabad, India
}

During this unprecedented time of the coronavirus disease 2019 (COVID 19) pandemic, most countries are struggling to optimize their healthcare resources. Although the prevention and treatment of COVID 19 has become the priority for the majority of the population, most medical professionals are facing the challenge of balancing the management of this pandemic and treating patients with other diseases. Healthcare professionals from all specialties are facing this situation and designing guidelines and recommendations that are contributing to the literature that is crucial for disease management at the current time. We share our experience with two ends of the spectra that we currently observe in spine patients. One group of patients included the non-operative cases that presented with back pain. Further, we discuss our experience with operative precautions and digitally assisted discharge.

Keywords: Orthopedic; Spine; Trauma; COVID 19; Coronavirus

\section{Introduction}

During these unprecedented times of coronavirus disease 2019 (COVID 19) pandemic, most countries are struggling toward the goal of optimizing healthcare resources. Although the prevention and treatment of COVID 19 has become the priority for the majority of population, most medical professionals are facing the dilemma of balancing out essential healthcare for patients suffering from other diseases.

Medical care personnel from all specialties are facing this situation and have established guidelines and recommendations that have contributed to the literature and are providing guidance for managing the pandemic. Here, we share our experience with two ends of the spectra that we are currently observing in spine patients.

\section{Non-operative Cases}

Most countries, including India, are on various degrees of lockdown, and the majority of the population has been spending time inside their homes since few months at this point. This situation raises the issue of an essentially sedentary lifestyle, and a subset of people, essentially youngsters, are spending their time in social media use on their mobile phones and in watching television and using the laptop. This situation, combined with the abnormal posture resulting from this excessive use of gadgets has

Received Apr 26, 2020; Revised May 7, 2020; Accepted May 7, 2020

Corresponding author: Awkash Kumar

Department of Orthopedics, Shalby Hospital, Ahmedabad, India

Tel: +91-8238034069, E-mail: awkash_raj@yahoo.co.in 
led to a surge in the cases of back pain reported to our telemedicine unit. Most of these patients are young adults who have active internet users.

Another subset of the back-pain patients reporting to us are those with increased household duties resulting from the absence of house help and raised workload because all family members are at home. Among these, elderly patients with osteoporosis and degenerative spine disease are reporting pain resulting from carrying kids in their arms that necessitates more forward-bending movements, given that they spend more time together.

Telemedicine consultants are issuing instructions that encourage the patients to consider a lifestyle change and include stretching exercises along with back exercises in the daily routine. Elderly patients are advised to avoid lifting kids or heavy weights, to adhere to their osteoporosis medication schedule, and to use supportive braces while performing their daily routine activities. Prevention and long-distance consultations are the biggest contribution to healthcare in the current time.

\section{Operative Cases}

Similar to the protocol being followed around the world, only emergency cases are being operated, and electives cases are being rescheduled. The operative requirement is being decided on a case-to-case basis by our medical team. Non-urgent cases that cannot be deferred for long are being marked as priority and are being scheduled for a status review telemedicine consultation.

Our focus is the prevention of infection spread to the operative team and staff during the surgery. One of the main concerns is aerosol production during anesthesia administration and surgery. Anesthetists are following their respective guidelines to minimize the aerosol exposure. We ensure that our surgical team enters the operative room at the precise time of their role in the surgery; unnecessary presence of team members is discouraged. It is mandatory for all the operation theatre personnel to wear the personal protection equipment (PPE) before starting the surgery. This PPE includes an N95 mask (National Institute for Occupational Safety and Health-approved mask), a disposable long sleeve waterproof gown, disposable double pair nitrile gloves, protective goggles, disposable head caps, and long shoe covers.

A standard procedure is being followed while removing the PPE to prevent recontamination. All the medical personnel are being trained and updated regularly as per the latest COVID-19 guidelines.

As per the existing study [1], aerosol production is usually the highest during the initial part of the surgery during surgical site incision using electrocautery, especially when accompanied by suction and irrigation. More blood during the procedure also contributes to more aerosol production.

We ensured aerosol minimization by using the following measures: (1) minimize blood in the field; (2) use of hypotensive anesthesia; (3) use of tranexamic acid before the surgery; (4) adrenaline infiltration; (5) chemical cautery using small $\mathrm{H}_{2} \mathrm{O}_{2}$ swabs (not to be used for posterior decompression or dural tear or when neural tissue is exposed) or adrenaline-soaked swabs in preference to electrocautery; and (6) ensuring smoke evacuation with high volume suction if using electrocautery.

If bleeding is present, (1) prefer mops instead of suction; (2) no use of water syringe jets; and (3) use of gauze or mops for wound packing.

In addition, other precautions, such as avoiding the use of power tools, such as burr and ultrasonic scalpel as far as possible, were also taken. Decompression was mainly performed by using bone rongeur and nibbler. We performed only one surgery in an operation theatre each day because blood-suspended aerosols do not settle down by cleaning. The operation theatres were cleaned in detail to improve the safety.

\section{Postoperative}

We ensured that the hospital stays of postoperative patients were minimized. The personnel that attended the patients were trained in wound care, and all patients with access to a smartphone were discharged earlier and diverted to the digitally assisted discharge team. These patients are being assisted in postoperative care using digital exchange of postoperative instructions and digital updates of clinical pictures of wound and range of motion. Further follow-ups were scheduled via tele-consultation, and patients are physically consulted at the hospital on a need basis.

Our experience revealed that we were successful in significantly minimizing unnecessary hospital visits and enhanced patient safety without compromising on patient care. 


\section{Conclusions}

Thus, minimizing unnecessary patient visits, issuing preventive instructions to predisposed patients, ensuring the safety of the operative team, are maximizing the use of digital media for postoperative care surveillance are vital in the ongoing scenario. We need to ensure that we do our bit to provide empathetic spine care to patients despite the ongoing pandemic.

\section{Conflict of Interest}

No potential conflict of interest relevant to this article was reported.

\section{References}

1. Yeh HC, Turner RS, Jones RK, Muggenburg BA, Lundgren DL, Smith JP. Characterization of aerosols produced during surgical procedures in hospitals. Aerosol Sci Technol 1995;22:151-61. 